\title{
Complete primary pachydermoperiostosis
}

\section{Nilusha Weerasooriya ${ }^{1}$, Amanda Amarasinghe ${ }^{1}$, Lakshmina Punniyamoorthy ${ }^{1}$, Harindra Karunatilake', Ananda Jayanaga ${ }^{1}$}

Ceylon Medical Journal 2020; 65: 118-119

DOI: http://doi.org/10.4038/cmj.v65i4.9283

\section{Introduction}

A 22-year-old Sri Lankan male presented to the National Hospital of Sri Lanka, with a six-year history of painful large joints involving bilateral knees, ankles and wrists. Examination revealed a young male with a normal physique and intelligence. He had grade IV clubbing with swollen ankle and knee joints with effusions (Figure 1). He had a coarse facial appearance due to hypertrophic scars caused by severe acne. There were thick oily skin and pronounced folds on his forehead, which is also known as cutis verticis gyrate (Figure 2). These features appeared gradually and were progressive. He also had seborrheic dermatitis on his chest. Mucosal and cutaneous examination of the rest of the body was normal.

Figure 1a.

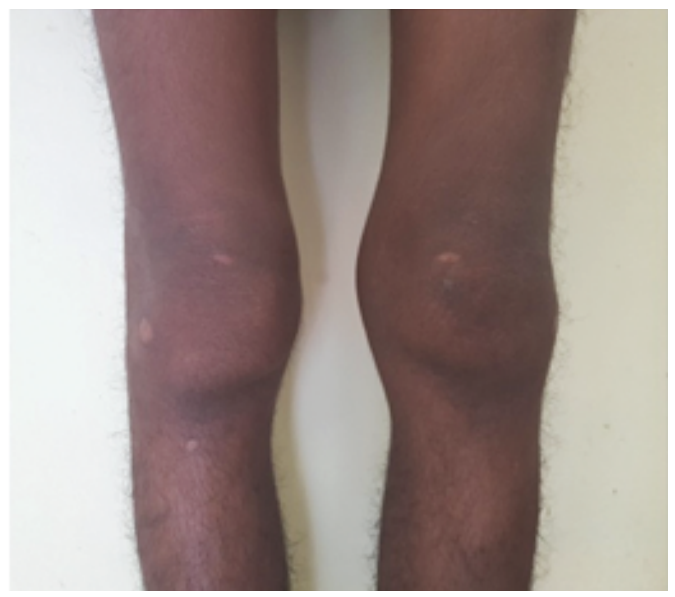

Figure 1c.

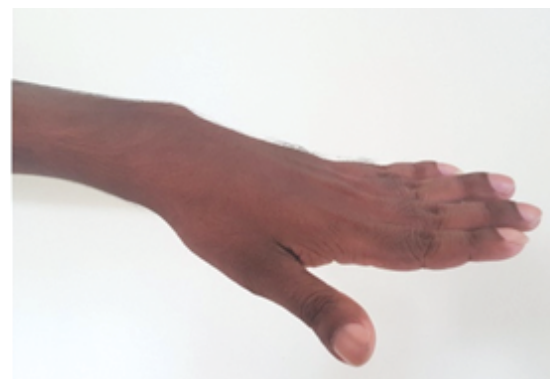

Figure 1b.

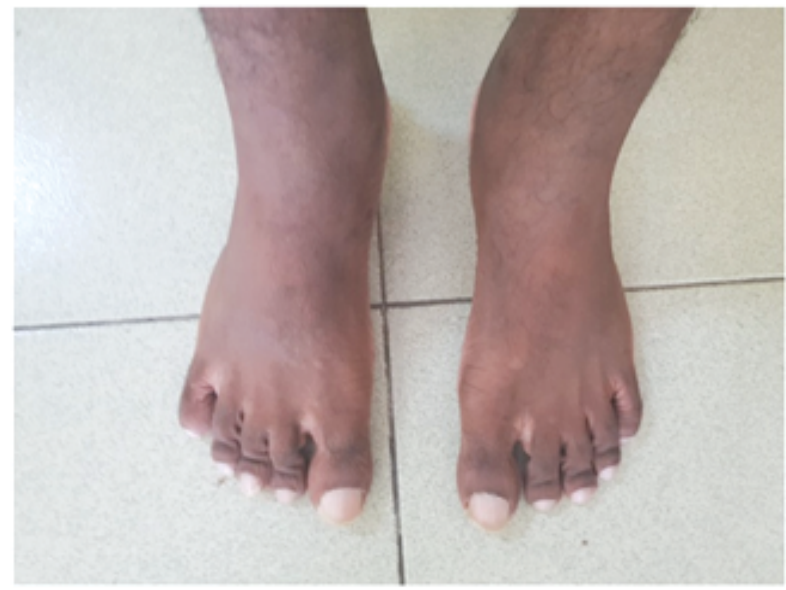

Figure 1d.

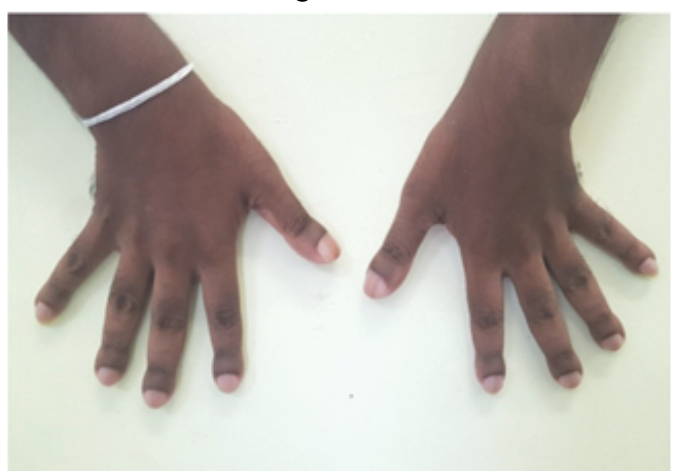

Figure 1. a) Swollen knee joints, b) Swollen ankle joints and toe clubbing, c), d) Finger clubbing.

${ }^{1}$ National Hospital of Sri Lanka, Colombo, Sri Lanka.

Correspondence: NW, e-mail: <wanilusha628@gmail.com>. Received 18 August 2020 and revised version 03 November 2020 accepted 02 December 2020.

This is an open-access article distributed under the terms of the Creative Commons Attribution License, which permits unrestricted use, distribution, and reproduction in any medium, provided the original author and source are credited. 


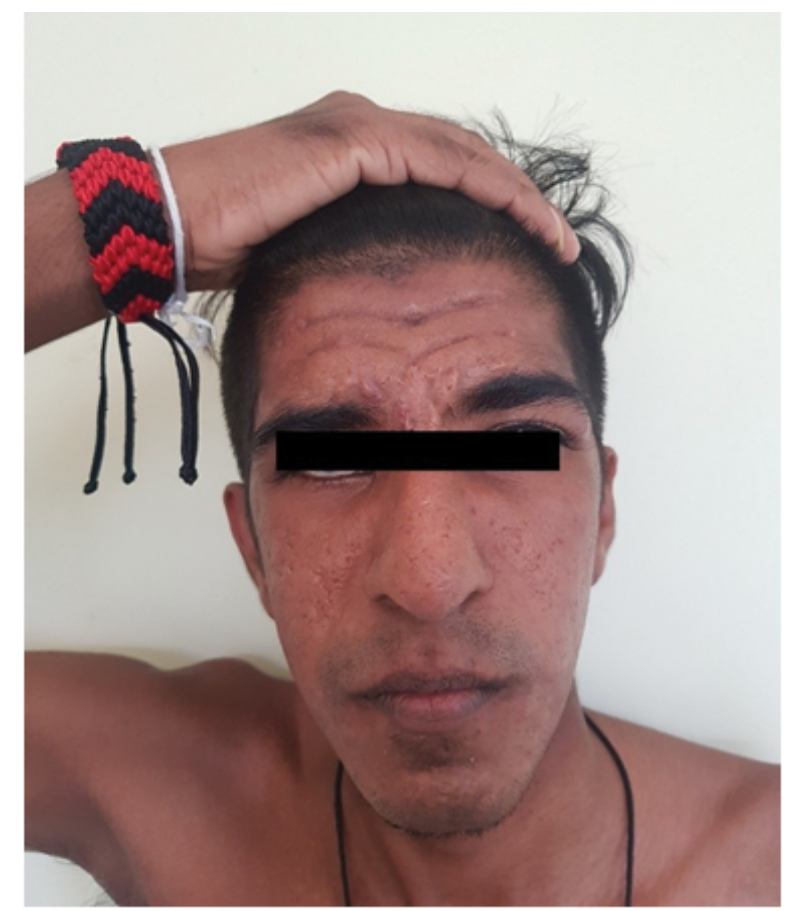

Figure 2. Acne and cutis verticis gyrate.

His laboratory investigations were normal. Plain $\mathrm{x}$ rays of long bones revealed a periosteal reaction with subperiosteal bone formation in the diaphysis (Figure 3). There was widening of the distal end of tibia and fibula at the ankle joints with marginal erosions and joint effusion (Figure 4).

The constellation of pachydermia, clubbing, and periosteal reaction in the long bones suggests a clinical diagnosis of hypertrophic osteoarthropathy (HOA) [1]. A search for an underlying heart, lung, liver, and intestinal disease to account for secondary HOA was negative. After ruling out the secondary causes of HOA, based on the clinico-radiological correlation, the diagnosis of the complete form of primary pachydermoperiostosis (PCP) was established.
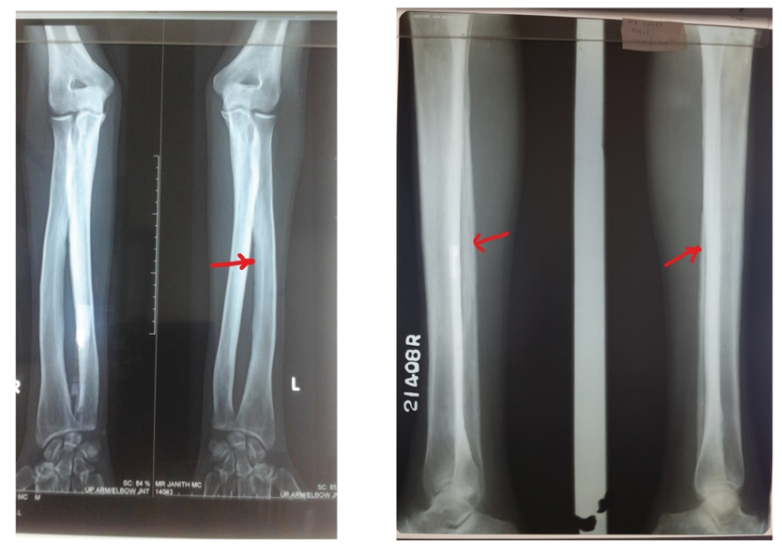

Figure 3. Arrows show periosteal reaction and subperiosteal bone formation.

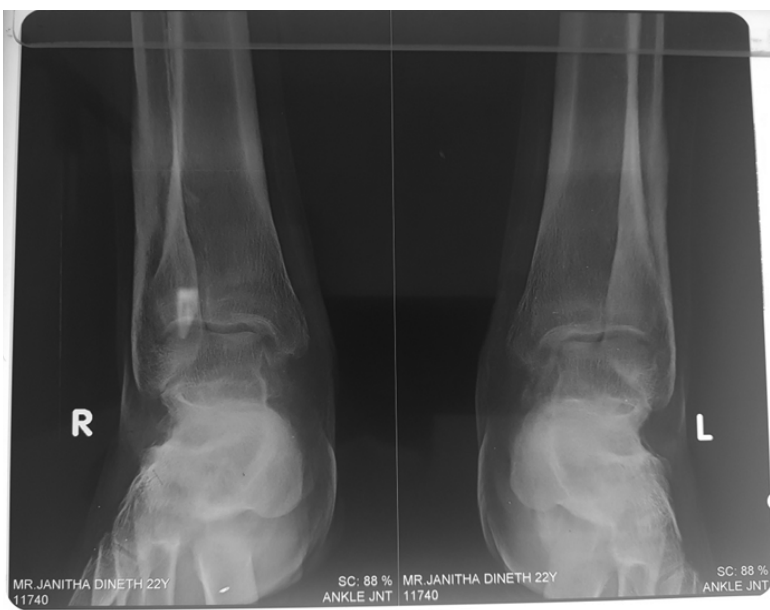

Figure 4. Widened ends of tibia and fibula with marginal erosions and joint effusion.

He was commenced on non-steroidal anti-inflammatory drugs, low dose steroids, and colchicine for arthropathy. Acne and pachyderma were treated with retinoids. The patient had a major cosmetic concern about his cutis verticis gyrate for which he is currently receiving botulinum toxin injection. At present, he is being followed up regularly in the medical, plastic, and reconstructive surgery and dermatology clinics.

$\mathrm{PCP}$ is a rare and important disease. The diagnosis of PCP requires a great deal of caution owing to its rarity and varied presentation. Practicing clinicians should be aware of the characteristic cutaneous manifestations of PCP and should be looked for in any patient with HOA. The family history of PCP should be sought after suspecting the diagnosis [2]. Although the management of primary PCP is supportive, patients should be followed up and monitored for complications of the disease as well as for the subsequent development of secondary aetiology $[3,4]$.

\section{References}

1. Touraine A, Solente G, Gole L. Un syndrome osteodermopathique: La pachydermia plicaturee avec pachyperiostose des extremites. Presse Med. 1935; 43: 1820-1824.

2. Rimoin DL. Pachydermoperiostosis (Idiopathic Clubbing and Periostosis). Genetic and physiologic considerations. N Engl J Med. 1965; 272: 923-31.

3. Ikeda F, Okada H, Mizuno M, et al. Pachydermoperiostosis associated with juvenile polyps of the stomach and gastric adenocarcinoma. J Gastroenterol. 2004; 39: $370 \mathrm{e} 374$.

4. Lowenthal MN, Tombak A, Lowenthal A. Secondary hypertrophic osteoarthropathy (HOA) mimicking primary HOA (pachydermoperiostitis or Touraine-Solente-Golé) syndrome. Israel Med Assoc J. 2004; 6(1): 64. 